\title{
A Rare Case of Acute Myocarditis
}

Karoly Peter Sarvari ${ }^{1 *}$, Szilard Zolyomi², Gergely Agoston ${ }^{2}$, Gabriella Terhes' ${ }^{1}$, Henriette Gavaller ${ }^{2}$, Tamas Forster ${ }^{2}$, Albert Varga ${ }^{2}$ and Edit Urban ${ }_{1}^{1}$ Insitute of Clinical Microbiology, Faculty of Medicine, University of Szeged, Szeged, Hungary

${ }^{2}$ Department of Internal Medicine and Centre of Cardiology, Faculty of Medicine, University of Szeged, Szeged, Hungary

\begin{abstract}
Acute myocarditis is an inflammatory disease with a quite broad range of consequences caused mostly by viruses. Epstein-Barr virus (EBV) rarely causes acute myocarditis. A 19-year-old healthy man with sudden epigastrial and chest pain was admitted to hospital. Laboratory investigations suspected acute myocardial infarct, but MRI and echocardiography showed myocardial oedema as part of inflammation. In his past medical history, toxic exposition and travelling abroad could not be recorded. PCR investigations of the most common viruses causing acute myocarditis gave negative results, but positive EBV anti-VCA IgM, and later sero conversion could be detected and simultaneously, a typical mononucleosis proved the aetiological role of EBV. After a 3-week-long high dose NSAID therapy on the basis of beta-blocker and ACE-inhibitor therapy, on control MRI oedema disappeared, but electrocardiography was still positive, the patient with satisfying general status was discharged home. On the 6 month control MRI, neither any signs of myocarditis, nor decreased systolic ejection fraction were noted, and also ECG turned negative.
\end{abstract}

Keywords: Acute myocarditis; EBV; Oedema; Electrocardiography and Laboratory investigation

\section{Why this Case is Important?}

Myocarditis is an inflammatory disease of the myocardium caused by a broad range of insults (e. g., infectious, autoimmune, toxic, drug-induced, hypersensitive and vasculitic) [1]. The spectrum of consequences of an acute myocarditis is quite wide; ranging from sudden death, Dilated Cardio Myopathy (DCMI), conductive disturbances and chronic heart failure [2]. Viral infection is the most prevalent aetiology, caused by usually enteroviruses, adenovirus, parvovirus B19, human herpes virus 6 , cytomegalovirus [3]. Although $90 \%$ of adult population are infected with Epstein-Barr virus (EBV), this virus rarely causes acute myocarditis [4].

\section{Case Report}

\section{Case description}

In March 2013, a 19-year-old man was admitted to the local Department of Internal Medicine with sudden epigastric pain spreading slowly on the chest. The pain disappeared during lying in the bed, but returned soon after. As the physical status, mild splenomegaly and lymphadenomegaly could be detected on the neck, but any other symptoms, including fever could not be demonstrated. Laboratory investigations gave the following results: elevated level of lactate-dehydrogenase ( $499 \mathrm{U} / \mathrm{L}$; reference: $<480 \mathrm{U} / \mathrm{L}$ ), aspartateaminotransferase $(66 \mathrm{U} / \mathrm{L}$; reference: $<37 \mathrm{U} / \mathrm{L})$, total creatine-kinase (603 U/L; reference: $195 \mathrm{U} / \mathrm{L})$, creatine-kinase MB (58 U/L; reference: $25 \mathrm{U} / \mathrm{L})$, and CRP $(5.2 \mathrm{mg} / \mathrm{L} ;<4 \mathrm{mg} / \mathrm{L})$. Diffuse ST-segment elevation and inverse T-wave on electrocardiography could be observed, on the basis of these findings, acute myocardial infarction was suspected (Figure 1), troponin- $\mathrm{T}$ was positive. Radiological examinations were performed (chest X-ray, chest multislice CT, coronarography and echocardiography) with negative results and stable haemodynamic parameters. Nevertheless, the suspition of myocarditis was risen, MRI showed oedema on the anterior, posterior and lateral wall and apex of the left ventricle (Figure 2). Relative lymphocytosis $(66.8 \%$; reference: $25-30 \%)$ and monocytosis (11.8\%; reference: $4-8 \%$ ) in the blood test referred to acute myocarditis, as well. He had no past medical history and was not on any medication. Toxic exposition could be excluded and he had not travelled abroad till admission. Because

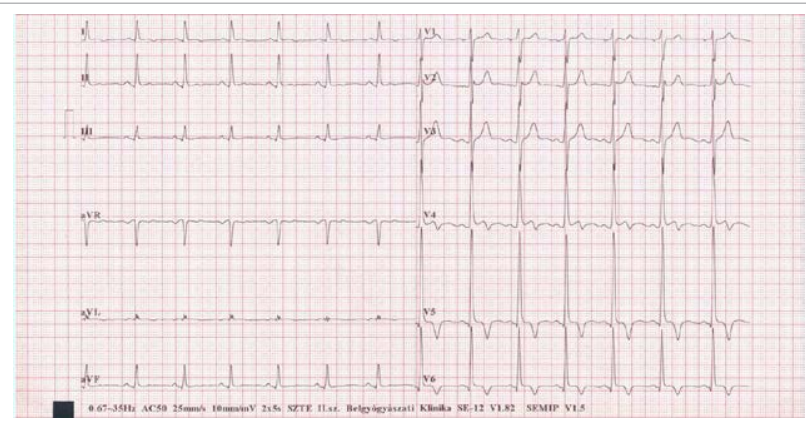

Figure 1: The ECG picture taken soon after admission shows ST- segment elevation in $\mathrm{V} 5$ and $\mathrm{V} 6$ and inverse T-wave.

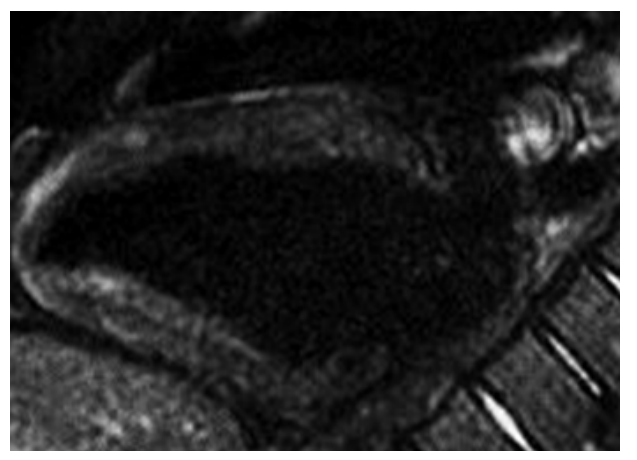

Figure 2: MRI shows oedema on the area of apex of the left ventricle.

*Corresponding author: Karoly Peter Sarvari, Insitute of Clinical Microbiology, Faculty of Medicine, University of Szeged, Szeged, Hungary, Tel/Fax: +3662545712; E-mail: sarvari.karoly.peter@med.u-szeged.hu

Received June 01, 2015; Accepted June 03, 2015; Published June 06, 2015

Citation: Sarvari KP, Zolyomi S, Agoston G, Terhes G, Gavaller H, et al. (2015) A Rare Case of Acute Myocarditis. J Med Microb Diagn 4: 193. doi:10.4172/21610703.1000193

Copyright: @ 2015 Sarvari KP, et al. This is an open-access article distributed under the terms of the Creative Commons Attribution License, which permits unrestricted use, distribution, and reproduction in any medium, provided the original author and source are credited. 
of the patient's satisfying general condition, myocardial biopsy was not performed. Real-time PCRs of the most frequent myocarditis-causing viruses (enterovirus and parvovirus B19) from plasma gave negative results. Serological examinations were also performed on admission for cytomegalovirus (CMV), Epstein-Barr virus (EBV), adenovirus and varicella. In the case of CMV serology, both CMV specific immunoglobulin $\mathrm{M}$ (IgM) and $\mathrm{G}$ (IgG) (DiaSorin, Germany) were negative, adenovirus specific IgM was also negative (Virotech/Sekisui, Japan), while adenovirus specific IgG was positive (Virotech/Sekisui, Japan). EBV specific anti-VCA IgM serology gave positive result, antiVCA and EBNA IgG serologies were negative (DiaSorin, Germany). In the case of EBV serology, seroconversion could be detected in the second sample one week after the first sample collection. On the other hand, during the acute myocarditis, the patient suffered from acute follicular tonsillitis as atypical infectious mononucleosis. After a 3-week-long high dose NSAID therapy on the basis of routine betablocker and ACE inhibitor medication, control MRI was negative, the patient was discharged home. On the 6 month-follow-up, MRI was still negative, no late signs of myocarditis wee observed, and also the systolic ejection fraction was preserved, control ECG returned to normal

\section{Other similar and contrasting cases in the literature}

To diagnose acute viral myocarditis is a very challenging task, because most of the cases are asymptomatic. According to the literature, acute myocarditis caused by EBV in immune competent patients has been rarely reported [5]. In a case report published by Ishikawa et al., a 17-year-old young woman died of sudden death caused by acute myocarditis due to EBV infection with cold-like symptoms and high fever for several days [6]. In another case report, a 24-year-old man was admitted to hospital with high fever, cough and epigastral pain. Infectious gastritis has been suspected and he was treated on the basis of this and three days later he was found dead in his home. Autopsy and PCR from the myocardium clarified the pathogenic role of EBV in acute myocarditis [6].

\section{Discussion}

Acute myocarditis presents multiple challenges both in the diagnosis and treatment [1]. The presentation of myocarditis can vary from asymptomatic electrocardiographic changes to fulminant heart failure. Acute myocarditis should be suspected whenever a young patient, presents with otherwise unexplained cardiac abnormalities, such as heart failure, cardiac arrhythmias, or conduction disturbances. Echocardiography helps to evaluate motion abnormalities, valvular function and pericardial effusion. MRI is a highly sensitive and specific method to detect characteristic changes in myocarditis, such as oedema, hyperaemia and cellular necrosis. Myocardial biopsy is recommended in acute heart failure unresponsive to treatment, giant cell myocarditis and eosinophil necrotising myocarditis [1]. Although the diagnostic value of viral serology is limited in this disease, because of the patient's satisfying general status, myocardial biopsy was not necessary [7]. The most common EBV-associated diseases are infectious mononucleosis,
Burkitt-lymphoma, nasopharyngeal carcinoma, Hodgkin-lymphoma etc., but very rarely-about $1 \%$ of all infectious cases- causes acute myocarditis.

We report here a case of myocarditis due to EBV infection. Prior to the hospital admission, the patient had no characteristic symptoms referring to EBV infections. The results of laboratory investigations (elevated liver enzyme and lymphocytosis) and microbiological examinations (detection of EBV specific immunoglobulins) confirmed the aetiological role of EBV [8]. MRI and electrocardiography showed changes referring to myocarditis. Because of the lack of toxic exposure and anatomic abnormalities, the possible pathogenic role of viruses had been arisen. Because of the otherwise good general condition of this patient, biopsy was not performed thus serological investigations were set up for the most important viral pathogens. This case may emphasize the possible pathogenic role of viruses in myocarditis in otherwise healthy persons and important role of serological investigations in this setting.

\section{References}

1. Shauer A, Gotsman I, Keren A, Zwas DR, Hellman Y, et al. (2013) Acute viral myocarditis: current concepts in diagnosis and treatment. Israel Medical Association Journal 15: 180-185.

2. Kaerney MT, Cotton JM, Richardson PJ, Shah AM (2001) Viral myocarditis and dilated cardiomyopathy: mechanisms, infections and management. Postgraduate Medical Journal 77: 4-10.

3. Mahfoud F, Gärtner B, Kindermann M, Ukena CH, Gadomski K, et al. (2011) Virus serology in patients with suspected myocarditis: utility or futility? European Heart Journal 10: 493-500.

4. Horowitz A, Zein I E, Valentiono R, Medhaoui H, Inamo J (2012) How an EbsteinBarr Virus may induce acute fulminant myocarditis in a young immuncompetent adult: a case report. West Indian Medical Journal 61: 640-642.

5. Roubille F, Gahide G, Moore-Moris T, Granier M, Davy J-M, et al. (2008) Epstein-Barr virus (EBV) and acute myopericarditis in an immunocompetent patient: first demonstrated case and discussion. Internal Medicine 47: 627-629.

6. Ishikawa T, Zhu B-L, Li D-R, Zhao D, Maeda H (2005) Epstein-Barr virus myocarditis as a cause of sudden death: two autopsy cases. International Journal of Legal Medicine 119: 231-235.

7. Kindermann I, Barth $\mathrm{CH}$, Mahfoud F, Ukena CH, Lenski M, et al. (2012) Update on myocarditis. Journal of the American Collage of Cardiology 59: 799-792.

8. Bowles NE, Ni J, Kearney DL, Pauschinger M, Schultheiss H-P, et al. (2003) Detection of viruses in myocardial tissues by polymerase chain reaction: evidence of adenovirus as a common cause of myocarditis in children and adults. International Journal of Legal Medicine 42: 466-472 